\title{
Diet Counselling - A Primordial Level of Prevention of Dental Caries.
}

\author{
Dr. Girish V Chour ${ }^{1}$, Dr. Rashmi G Chour ${ }^{2}$ \\ ${ }^{1}$ Department of Oral \& Maxillofacial Surgery, PMNM Dental College \& Hospital, Bagalkot, Karnataka, India \\ ${ }^{2}$ Department of Pedodontics \& Preventive dentistry, PMNM Dental College \& Hospital, Bagalkot, Karnataka, \\ India.
}

\begin{abstract}
Teeth are tools that have evolved to ensure survival of species. The tooth is the only hard organ in the biological kingdom that is comprised of two tissues of different embryological origin. It has evolved uniquely to fulfill its function. But even nature has to pay a price for creating so specialized grinding and cutting tool. Enamel tissue by virtue of its unique biological properties is highly susceptible to environmental agents. Change in behavior is a process affected by the established fact that humans, as well as animals, are neophobic. This means we have a fear of new things. Therefore, forced dietary changes cannot be successful unless the benefit accrues rapidly and is of demonstrable advantage. In addition to clinical service dietary activities, there is growing recognition of the importance of public health activities and efforts to disseminate culturally appropriate health promotional messages to help educate the public, health care professionals about the steps that need to be taken to enhance oral health for individuals and population at large. Hence dietary counseling in dental practice if not limited to sucrose restriction could therefore be beneficial in a community perspective as well. Hence the present article reviews about the importance of diet counseling in the prevention of dental caries.
\end{abstract}

Key words: dental caries, demineralization, diet counseling.

\section{Introduction:}

When man broke from the natural food chain, he developed new energy resources and applied technologies to food processing, since then our dietary habits have undergone major changes. Both the qualitative nature of our diet and pattern of eating has changed and are changing. ${ }^{1}$

Ingestion of food may affect oral-dental health by both systemic and local mechanisms. Nutritional effects are mediated systematically and the dietary effects are mediated locally in the oral cavity. The systemic effects result from the absorption and circulation of nutrients to all cells and tissues and may be mediated through influences on development of teeth, the quality and quantity of salivary secretion, improved host resistance and improved function. Dietary constituents exert their local effects by influencing the metabolism of the oral flora and by modifying salivary flow rates and, in-directly, the qualitative aspect of salivary secretions. Also important is the manner in which food items affect taste perception and condition dietary preferences and patterns of eating. ${ }^{1}$

\section{Caries as Nutritional Deficiency}

Some researches consider caries as nutritional deficiency caused either by sufficient phosphate intake or improper dietary calcium -phosphate ratio. None of these theories have adequate statistical or experimental support and hence these theories remain primarily conjectural.

Numerous experiments on rodents have demonstrated that phosphate salts have the potential to retard dental caries. It is not surprising, therefore, that several theories have been proposed dealing with the role of phosphate in the process of caries formation. Using radioactive phosphate, Luoma ${ }^{9}$ showed that inorganic phosphate was taken up by plaque bacteria during metabolism of carbohydrates, the phosphate being required for phosphorylation of sugars and for polyphosphates that store energy. It has been postulated that steady-state equilibrium exists between the inorganic phosphate of saliva and mineral phase of enamel. According to the phosphate sequestration theory, as bacteria take up phosphate, inorganic phosphate must be removed from enamel. However, in vivo, there is a continual flow of saliva containing soluble inorganic phosphates that are more readily available to bacteria than the insoluble mineral phase of enamel, provided the saliva can diffuse through the plaque to the bacteria. ${ }^{2}$

Alternative explanations consider caries as a nutritional deficiency caused either by insufficient phosphate intake or by an improper dietary calcium-to-phosphate ratio ${ }^{3}$. Neither of these latter explanations has adequate statistical or experimental support, and both remain primarily conjectural. 


\section{Current Concepts of Caries etiology}

The current concept considers caries as a dynamic and reversible process. Caries activity is the result of the interplay of a number of aetiological factors. Some of these factors cause demineralization; others promote remineralization of the tooth. Demineralization occurs several times a day following each period of food consumption. Usually demineralization is limited and quickly reversed by the effect of fluoride and the buffering and repair actions of saliva. Where this does not occur, progressive demineralization causes porosities to develop in enamel, forming the 'subsurface' incipient of early lesion. If this demineralization process continues, Cavitation will eventually result. Caries can be arrested or even reversed at the recavitated stage, providing a balance towards remineralisation can be established. The recognition of the fact that caries is potentially reversible in its early stage has considerable implications. ${ }^{4}$

One of the recent methods of describing the etiology of dental caries was put forth by Thylstrup and Fejerskovas ${ }^{4}$ :

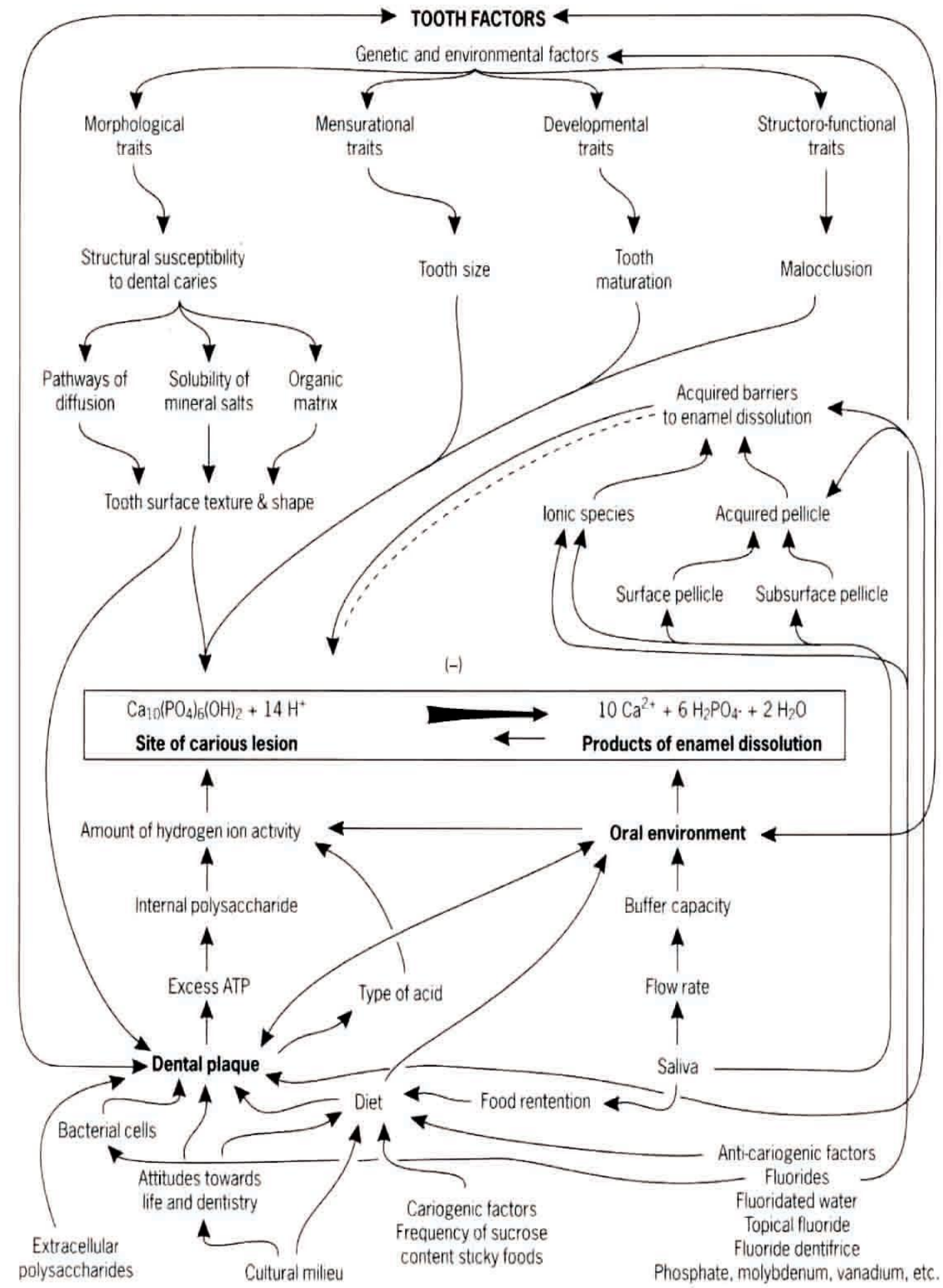




\section{Concept of Caries Balance}

The chronic nature of caries stems from the notion that dentate individuals remain at risk for the development of caries throughout their lifetimes. Individuals or populations who do not effectively balance the negative effects of prevailing risk factors (which can change over time) with adequate protective factors experience the clinical consequences of caries, which include diminished oral health and threats to general health from the spread of infection to adjacent or distant body structures. ${ }^{4}$

\section{Dynamic Balance between \\ Risk Factors \& Protective Factors}

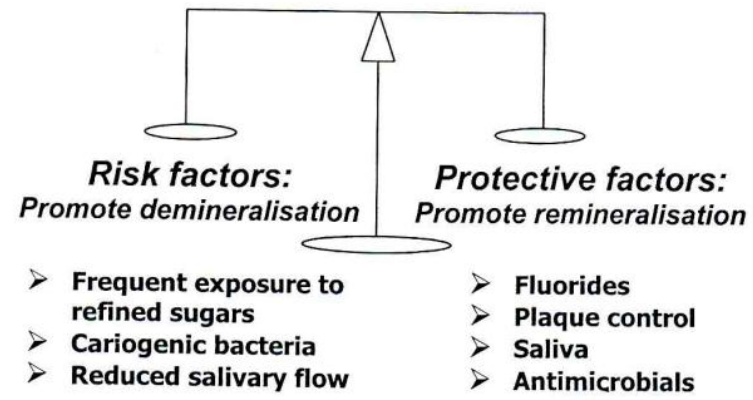

\section{Dynamic Balance between Risk Factors \& Protective Factors}

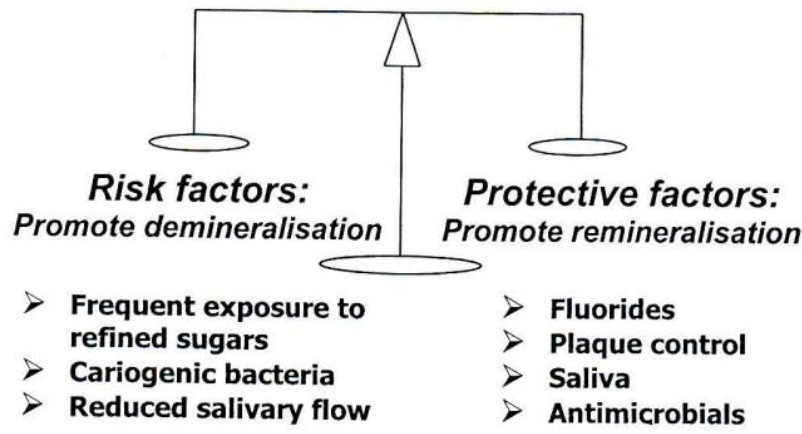

\section{Importance Of Diet And Nutrition In Pediatric Dentistry}

Optimal growth and development are the primary objectives of pediatric nutrition. A child's diet, defined as the combination of foods consumed and the nutrients contained therein has the profound ability to influence cognition, behavior and emotional development in addition to ultimate physical growth and development. Food is merely a vehicle for nutrient delivery; the nutrients provide energy for growth, serve as structural components, and participate in all metabolic functions of the body. Food, however is more than just nutrients : sensory , emotional , social and cultural associations influence food choices. ${ }^{5}$

\section{Dietary screening and education in the dental practice}

Dental professionals should routinely screen patients to assess the role of diet in caries risk and management. Dentists are not trained to conduct a complete nutritional assessment that includes anthropometries and biochemical data but they can use dietary screening, assessment, and analysis to provide nutrition and dietary education and referral to registered dietitians for more in-depth nutrition counseling. Screening activities should include assessment of determinants of dietary intake and behaviors that are associated with dental health status and caries risk. Rather than labeling foods as "allowable" or "to be avoided," nutrition messages need to be offered that promote health but respect the strong influence that taste has on food choices. $^{2}$ 


\title{
Objectives of Counseling
}

The main objective of dietary counseling in pediatric oral health is caries prevention. If prevention is indeed the objective, then diet assessment and preventive recommendations must begin at an early age, prior to visible signs of the carious process.DietCounselling aims to help parents change their and their children's dietary behaviours so that they choose diets with low or noncariogenic snacks, limit sweet foods to mealtimes and perform tooth brushing after sugar exposures. Thus in short the objectives of diet counselling are

1. Correction of diet imbalance, that could affect the patients general health and sometimes reflect on his oral health.

2. Modification of dietary habits, particularly the ingestion of sucrose containing foods in forms, amt, and circumstances that cause caries formation.

3. Dietary recommendations must be realistic and always based on current dietary behaviours of the family.It is pointless to prescribe changes that a patient cannot or will not implement

Additionally, modifications to the diet can only be made over time, aided by repitition and reinforcement.The goal must be to help caregivers develop lifelong dietary habits, which promote general and oral health for themselves and for those whom they influence.

\section{Art of Counseling}

$>$ Counseling is ONE-ONE basis.

$>$ To be effective it should be in form of dialogues. e.g.:- Communication technique:

$>$ During a Face-face interview, keeping eye contact with the patient is persuasive and powerful device for motivating behavioral change

$>$ Communication can be both verbal and non-verbal. Words transmit information. The interviewer's tone of voice, facial expression and gestures convey sincerity, enthusiasm, and empathy. The nonverbal actions can be influential in helping the patient to change his or her behavior.

$>$ Messages must be adopted to pts needs and level of understanding.

$>$ To communicate with a patient, a combination of interviewing, teaching, counseling and motivating is used. $^{14}$

\section{Basic Requisites Of Diet Counselling}

A basic requisite for accomplishing dietary change is the advice that the patient not the counselor bears the responsibility for making the change. The counsellor bears the responsibility for making the change .The counselor should explain candidly the need for full cooperation and a sincere effort by the patient to modify the diet, and the patient should agree.

\author{
A Model of Prenatal Counseling \\ Purpose \\ $>\quad$ To educate parents about dental development of the child \\ $>\quad$ To educate parents about dental disease and prevention \\ $>\quad$ To provide a suitable environment for the child \\ $>\quad$ To strengthen and prepare the child and dentition for life \\ Methods \\ $>\quad$ Education concerning development, prevention, and disease \\ $>\quad$ Demonstration of oral hygiene procedures \\ $>\quad$ Counseling to instill preventive attitudes and motivation \\ Content \\ Evaluation of learning, acceptance, and needs

\section{External Component (Parents)} \\ $>\quad$ Parents' education concerning dental disease and oral hygiene \\ $>\quad$ Parents' motivation for plaque removal program Changes in mother's oral health ,intake of \\ sweets\& Pregnancy gingivitis \\ $>\quad$ Myths and misconceptions about pregnancy and dentition Parents' dental treatment
}

\section{Internal Component (Parents and Child)}

$\begin{array}{ll}> & \text { Parents' education-development of child } \\ > & \text { Effect of lifestyle on child } \\ > & \text { Intake of sweets } \\ > & \text { Exposure to disease (e.g., rubellal syphilis) } \\ & \text { Effect of drugs on child-e.g., tetracyclines }\end{array}$


Nutrition

Calcium

Vitamins

Fluoricles

Essential nutrients

Child's needs after birth

Breast feeding versus bottle feeding

Fluoride supplementation

Teething

Hygiene

Non-nutritive sucking

First visit

During prenatal counseling, teething should be mentioned because it most likely will be the first oral event that parents have to deal with. Although the timing of eruption of teeth can usually be predicted, its occurrence frequently surprises new parents, and if the infant experiences difficulties it can create parental anxiety. Teething is a natural phenomenon that usually occurs with no problems. Nevertheless, some infants exhibit signs of systemic distress, including a rise in temperature, diarrhea, dehydration, increased salivation, skin eruptions, and gastrointestinal disturbances (Honig, 1975) ${ }^{8}$. Lancing of tissues is usually not indicated. Increased fluid consumption, a nonaspirin analgesic, and palliative care consisting of teething rings to apply cold and pressure to the affected areas generally reduce the symptoms and result in a happier infant (King, 1994). If symptoms persist for more than 24 hours, the infant should be examined by a physician to rule out upper respiratory infection and other common diseases and conditions of infancy.

Prenatal counseling programs also should provide guidelines for the parents about the timing of the first professional dental visit. Historically, asymptomatic children were scheduled for their first dental examination between 3 and 5 years of age. More recently, programs designed to provide comprehensive preventive measures have stressed the importance of initiating professional visits at an earlier age, at or shortly after the time of the eruption of teeth (Nowak, 1997).

Bright Futures - Guidelines for Health Supervision of Infants, Children, and Adolescents (Green, 1994) is a comprehensive and practical resource designed to assist health professionals and families to more effectively promote the health and well-being of all children and adolescents. It recommends an initial dental appointment at around 12 months of age to assess the infant's risk for dental disease, complete a clinical examination, provide anticipatory guidance information, and schedule a follow-up appointment (Nowak and Casamassimo, 1995) ${ }^{7}$.

Another innovation in designing preventive programs centers around the concept of risk assessment. The assessing of the child's risk by the dentist allows the practitioner to individualize oral health supervision. All children are not equally affected by dental disease. By determining risk factors, the dentist can individualize a preventive plan with protective factors that promote oral health. Risk factors can change over time. Therefore, the dentist must assess the status periodically and make appropriate modifications (Casamassimo, 1996).

\section{Diet counseling - discussion}

The patient selectionFive ' $W$ ' and one ' $H$ ' of diet consultation.

6 questions are to be made before making decision about which pts will benefit from diet counseling WHO, WHAT, WHY, WHEN, WHERE AND HOW.

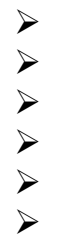

WHO may be benefited?

WHAT are the objectives of diet and nutrition counseling?

WHY is counseling beneficial?

WHEN is counseling conducted?

WHERE should the counseling occur?

HOW to counsel?

When providing dietary counseling in pediatrics, identification of the "patient "requires careful consideration. Diet counseling will not succeed with every dental patient. Persons who need counseling must also want information about their potential dental caries problem and must be willing to improve current undesirable food selections and eating habits. Potential candidates for counseling should give high priority to preventive dentistry and should be willing to expend long term efforts to maintain their natural dentition in good health for a lifetime. ${ }^{6}$

\section{The Dental Health Diet Score}

The dental health diet score is a screening device to achieve this objective .It is a simple scoring procedure that can disclose a potential dietary problem that is likely to adversely affect a patient's dental health. ${ }^{6}$ 
Essentially, the dental health diet score gives points earned as a result of an adequate intake of food from each of the food groups plus points for ingesting foods especially recommended because they are the best sources of the ten nutrients essential for achieving and maintaining dental health .From this sum points are substracted for frequent ingestion of foods that are overtly sweet - whose sweetness is derived from added refined sugar or concentrated natural sugars.The difference is the dental health diet score.

A score of 60 to 100 is acceptable and dietary counseling is not usually given unless the patient requests it.If the score is 56 or less, dietary counseling is both indicated and recommended as a part of a comprehensive preventive dentistry service to patients.

\section{Instructions for calculating a dental health diet score Step 1(food score)}

To ascertain the average daily intake, list everything you eat and drink on an ordinary weekday including snacks. Record the time when the meal or snacks were eaten, the amount ingested, how the food was prepared, and the number of teaspoons of sugar added

\section{STEP 2}

Circle the foods in the diary that have been sweetened with added sugars or are concentrated natural sweets. Classify the uncircled foods or mixed food dishes into one or more of the appropriate food groups For each serving of these foods listed in the food intake dairy, place a check mark in the appropriate food group block.

Add the number of checks and multiply by the number shown .The maximum, number of points credit for the milk and meat group is 24 each and for the fruit, vegetable and the bread cereal group is 24 each Add the points .

The sum is the food group score

This is to find out how much of the nutrients are present in the food taken. Check the food in each column then, circle the number 7 besides the nutrients that head the column. Only 7 points are given per nutrient. Add the circled numbers. ( Perfect score-56 )

\section{Step IV (Sweet Score)}

List the sweet, sugared foods, and the frequency with which they are consumed. Classify each sweet into liquid, solid and sticky or slowly dissolving. Place check mark in the frequency column of each item as long as they are eaten 20 min apart. Add the checks- if sweets are liquid -x5, solid- x10,slowly dissolving- x15.

(Highest Score-35)

\section{StepV}

All the food group scores and sweet scores summed to the totaling score.

If the food scores is barely adequate or inadequate or sweet score is "watch out" zone.-NUTRITIONCOUNSELING is required.

4 food group scores

72-96 -----Excellent

64-72 -----Adequate

56-64 -----Barely adequate

56 or less -----Not adequate.

Score 60-100 is acceptable, and diet counseling is given only at pt request. If 56 or less ,then dietary counseling is both recommended and indicated as a part of preventive program. ${ }^{6}$

Dietary screening and assessment questionnaire for dental settings

Part A of the questionnaire includes those dietary behaviors that were most predictive of caries risk. When responses to these are positive, part B can be initiated to identify additional information specific to understanding underlying issues that can be addressed in a targeted nutrition message. When responses to these questions are combined with assessment of oral health status, xerostomic potential, and denial treatment needs, tailored messages evolve that help patients recognize the importance of oral health within the scheme of achieving overall health status. By way of this approach or one similar, the dental professional is better prepared to offer nutrition education massages consistent with dental research findings and health promotion guidelines. ${ }^{2}$

\section{Dietary advice :}

Giving dietary advice successfully, therefore depends on for more than providing knowledge, it requires sensitive understanding of the role of food in society and its significance for the individual patients. Dietary advice consists of several distinct elements which cannot be considered as one of the events. Changing eating habits is one of the most difficult changes in behavious to achieve because of all pervasive nature of food. 
Ideally, changes in food related behavious should be paralleled by changes in other aspects of health related behavious in order to produce healthier person in all respects. ${ }^{6}$

\section{Conclusion :}

Dental caries is a diet related disease that continues to be a problem for certain dental patients. The dental team should thoroughly understand the relationship of diet to caries and conscientiously apply that knowledge to educate the patients in general as well as counsel special high risk individuals. ${ }^{5}$

Recent advances in our understanding of nature of caries and scientists oblige to develop more refined strategies for addressing the fundamental aspect of the caries process have stimulated a host of new anti caries research and development activities.

In the mean the evidence from epidemiologically great studies suggest that readily available relatively low thin approaches include minimizing exposure to refined carbohydrates plaque control measures and optimal exposure to various forms of fluoride on an ongoing basis.

Exposure to refined carbohydrates is a function of personal life style and the availability of various food options. Since dietary habits are to personal, dietary adult must be personalized if it is to be successful. ${ }^{4} \mathrm{~A}$ parallel to this is the well - known fact in the food industry that food items with the label 'NEW' do not sell, but those with a traditional look do. It is also important that the advice is compatible with medications and diseases of the individual patient, and that the proposed changes are acceptable. Of further importance is that such counsel takes account of the social situation of the patient. ${ }^{2}$

\section{Refernaces}

[1] Nikiforoik - Understanding dental caries (part I) P - 60- S. Karger A.G 1985.

[2] Thylstrup A, Fejerskov. Text book of clinical cariology $2^{\text {nd }}$ Edition p: 283 - Munksgaard 1994.

[3] Rugg Gunn and H. Nunn - Nutrition diet and oral health p : 27-9.Oxford Medical Publication 1999.

[4] Crall J J. Optimising oral health throughout childhood : The importance of caries risk assessment and strategic interventions. International Dental Journal 2007, 57, p : 221-228.

[5] Laura M. Romito - Nutrition and Oral Health Dental clinics of North America, April 2003; 47 No.2 p : 187-207, 279-303 WB . Saunders.

[6] Nizel papas. Nutrition in clinical dentistry, $3^{\text {rd }}$ edition P: 277-307 WB saunders and company 1989

[7] MC Donal Avery Dean Dentistry for child and adolescent. Eight editions, Mosby 2004. Dentistry for the child and adolescent p : 266-267

[8] Zero D.T. Sugars - The arch criminal. Caries Res $2004 ; 38: 277-285$

[9] Rugg - Gum AJ, Hackett AF, Appleton DR, Jenkens GN, Eastoe JE. Relationship between dietary habits and caries increments assessed over 2 years in 405 English adolescent young children. Arch oral Biol1984;29 : 983-992. 